\title{
Oxygen Reduction at Palladium Decorated Copper-Molybdenum Oxide Modified Glassy Carbon Electrode
}

\author{
K. Volkan Özdokur* \\ Department of Chemistry, Faculty of Science and Letter, Erzincan Binali Yıldırım University, Erzincan, Turkey \\ *vozdokur@gmail.com
}

Received: 20 February 2019

Accepted: 12 September 2019

DOI: $10.18466 /$ cbayarfbe. 529847

\begin{abstract}
In this study, metal nanoparticles decorated metal oxide electrode was fabricated via electrochemical technique. Firstly, molybdenum/copper oxide was electrochemically deposited on the bare glassy carbon electrode surface. Then palladium nanoparticles were modified on this oxide functionalized surface by consecutive potential cycles. Resulting composite electrode was characterized with scanning electron microscopy. The proposed composite electrode was employed for electrocatalytic oxygen reduction in alkaline media. As compared to bare electrode, the resulting composite surface has tremendous effect on oxygen reduction in terms of accelerated peak current values.
\end{abstract}

Keywords: Oxygen reduction, Pd nanoparticles, Molybdenum and Copper oxide.

\section{Introduction}

Oxygen reduction reaction (ORR) is one of the most studied electrochemical reactions due to its importance in energy field, especially in fuel cells [1].The reduction of oxygen to water can occur via two parallel pathways that are highly influenced by the electrode material. In two step-two electron pathway that known as "peroxide route" occur with two electron reduction of oxygen to hydrogen peroxide. The other is reduction of molecular oxygen to water through a four-electron pathway [2]. The electrochemical behavior of oxygen reduction has been studied in alkaline, neutral, and acidic media [3-4].

Catalytic activity of the electrode mainly depends on its composition that is significant to design effective component for electrode modification. For this purpose various modifiers were employed such as carbon nanotubes [5], polymers [6], phthalocyanines [7], metal nanoparticles [8] and metal oxides [9]. In this study, we focus on the metal oxide and metal nanoparticles as electrode modifiers towards ORR.

Metal oxides comprised a significant group of versatile material for electrocatalysis with their high electronic conductivity and stability. Lower costs of metal oxides relative to platinum catalysts make them preferable for many applications. Several studies reported the use of metal oxides as sensitive electrodes for some analytes [10-11], and also oxygen reduction reaction [12].

Metal nanoparticles (MNPs) were also another group of modifier which facilitate electron transfer rate and increase the active surface area of electrode. Pd is one of the most widely used metals among all MNPs due to its ability to promote electrode reaction [13] that is why it employed by various researchers [14-15].

In this paper, molybdenum/copper oxide $(\mathrm{MoOx}-\mathrm{CuOx})$ nanostructure supported on glassy carbon electrode surface (GCE/MoOx-CuOx) using a simple and practical method. And then $\mathrm{Pd}$ nanoparticles were modified on this surface by electrochemical technique (GCE/MoOx-CuOx). Electrocatalytic oxygen reduction reaction was investigated in alkaline media and satisfactory peak enhancement was obtained with the proposed electrode.

\section{Materials and Methods}

\subsection{Reagents and Apparatus}

Sodium molybdate dihydrate $\left(\mathrm{Na}_{2} \mathrm{MoO}_{4} \cdot 2 \mathrm{H}_{2} \mathrm{O}\right)$, copper sulfate, sulfuric acid, acetic acid were purchased from Carlo Erba. $\mathrm{NaOH}$ was purchased from Riedel de Haen. Palladium acetate was purchased from Aldrich. All solutions were prepared using ultrapure water (Milli-Q $18.2 \mathrm{M} \Omega \mathrm{cm}$, Millipore System Inc.).

A GAMBRY 3000 was used for voltammetric studies. All measurements were carried out in a three-electrode system consisting of; a platinum wire as a counter electrode, $\mathrm{Ag} / \mathrm{AgCl} / \mathrm{KCl}$ as a reference electrode, and working electrode (bare GCE, GCE/MoOx-CuOx and GCE/MoOx-CuOx/Pd). Modified electrodes were characterized with scanning electron microscope (SEM; FEI QUANTA 450).

\subsection{Preparation and Characterization of GCE/MoOx-CuOx/Pd}

The bare GCE was initially freshly polished and the GCE/MoOx-CuOx/Pd was prepared in two steps; at 
first, electrode was immersed into the cell containing $0.001 \mathrm{M} \mathrm{CuSO}_{4}$ and $0.01 \mathrm{M} \mathrm{Na}_{2} \mathrm{MoO}_{4}$ in $0.01 \mathrm{M} \mathrm{H}_{2} \mathrm{SO}_{4}$ solution. The $\mathrm{CV}$ program was applied between the potential of $-0.75 \mathrm{~V}$ to $0.8 \mathrm{~V}$ for 20 cycles (Fig.1a). The resulting electrode depicted as GCE/MoOx-CuOx. Then resulting electrode was immersed in $0.01 \mathrm{M} \mathrm{Pd}^{2+}$ containing $0.01 \mathrm{M} \mathrm{HCl}$ solution. A $\mathrm{CV}$ process was applied for 15 deposition cycles in the $-0.5 \mathrm{~V}$ to $0.7 \mathrm{~V}$ potential ranges (Fig.1b). Following electrode abbreviated as GCE/MoOx-CuOx/Pd. All modified electrodes were rinsed thoroughly with ultrapure water prior to use and voltammograms were measured at a scan rate of $50 \mathrm{mV} \mathrm{s}^{-1}$.
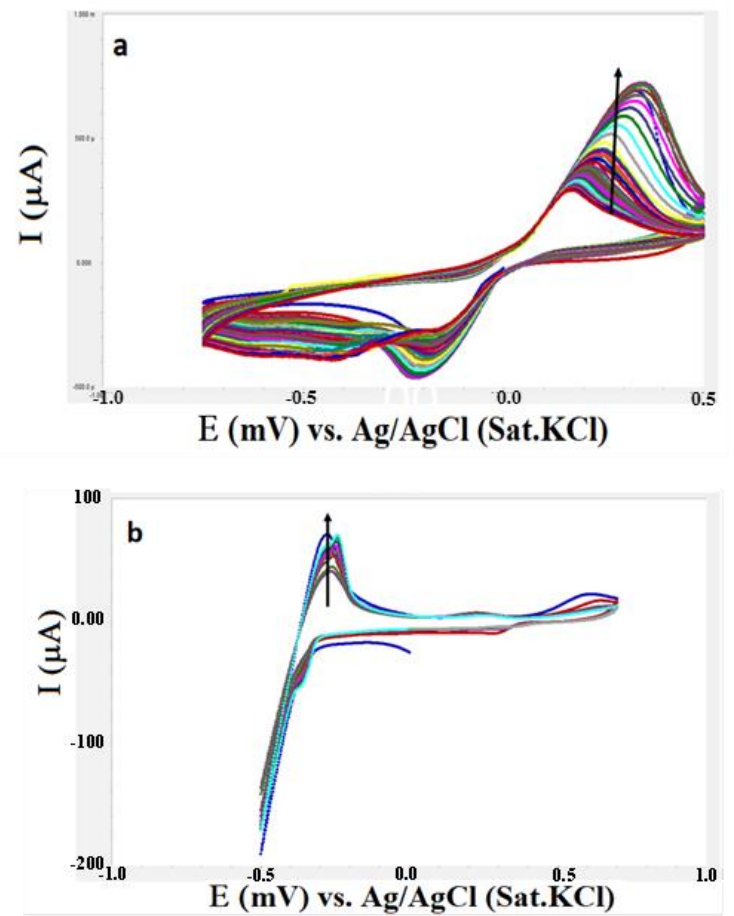

Figure 1. $\mathrm{CVs}$ of a) $\mathrm{MoOx}-\mathrm{CuOx}$ and b) $\mathrm{Pd}$ nanoparticles deposition.

\section{Results and Discussion}

According to the Fig.1a two reduction peaks were occurred at -0.2 and $-0.45 \mathrm{~V}$. The peak currents were increased with increasing cycle number and cathodic peak potential shift was observed for these peaks. The peak at $-0.2 \mathrm{~V}$ was associated with $\mathrm{Cu}^{2+}$ reduction [16] and the peak at $-0.45 \mathrm{~V}$ can be attributed to MoO42reduction [17] at the electrode surface. The potential shift at both peaks indicating the deposition of the $\mathrm{Cu}$ and Mo. The peak at $+0.25 \mathrm{~V}$ can be attributed oxidation of $\mathrm{Cu}$. The deposition of $\mathrm{Pd}$ nanoparticles took place at $0.3 \mathrm{~V}$ [18] and increased with the increasing cycle number. The peak at -0.3 in anodic direction can be attributed to hydrogen adsorption/desorption peaks.

Characterization of the electrode surface utilized with scanning electron microscopy (SEM). Smooth surface was obtained for bare GCE (Fig.2a) while obviously different structure formed with presence of $\mathrm{MoOx}$ -
$\mathrm{CuOx}$ (Fig.2b). MoOx-CuOx particles were decorated on the GCE surface with grape-like structure. Moreover, Fig.2c represents the Pd nanoparticles on GCE in order to understand the exact difference between modified surfaces. And finally GCE/MoOx-CuOx/Pd surface was observed at Fig.2d with aggregate like structures. EDX result of $\mathrm{GCE} / \mathrm{MoOx}-\mathrm{CuOx} / \mathrm{Pd}$ also confirms the presence of $\mathrm{Mo}, \mathrm{Cu}$ and $\mathrm{Pd}$ content with a $5.4 \%, 5.3 \%$, and $1.2 \%$ weight gain, respectively.

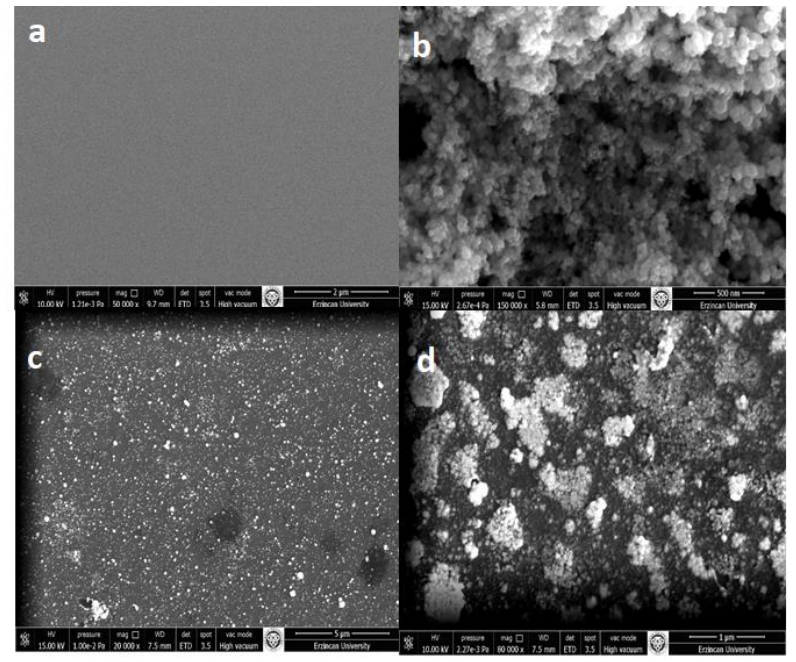

Figure 2. SEM images of a) Bare GCE, b) GCE/MoOx$\mathrm{CuOx}$ c) GCE/Pd and d) GCE/MoOx-CuOx/Pd.

In order to calculate the electroactive surface area (ESA) of electrodes, CV with a ferrocyanideferricyanide redox couple is generally used. CVs were recorded at various scan rates in a ferro-ferricyanide redox couple-containing solution; the square root of the scan rate versus the peak current was then plotted. The ESA can be calculated based on the Randles-Sevcik equation as follow the previously published literature [19]. Scan rate studies were conducted for bare and modified electrode $\left(10-350 \mathrm{mV} \mathrm{s}^{-1}\right)$. From the slope of the linear graph line, the electroactive areas of the GCE and GCE/MoOx-CuOx/Pd were calculated as 0.056 , and $0.1 \mathrm{~cm}^{2}$. These results confirm the improved activity of GCE/MoOx-CuOx/Pd towards ORR.

\subsection{ORR Investigation with Voltammetry}

In order to improve the activity of electrode, modifiers can be optimized. Therefore, cycle number of both Mo$\mathrm{Cu}$ and $\mathrm{Pd}$ were studied with increasing values to obtained best results towards ORR. Cycle number of Mo-Cu was varied between 2 to 10 and electrode signal was exhibited in Fig. 3a. Fig 3c shows the effect of cycle number on the ORR peak currents with column graph. 5 number of cycles was chosen as an optimum due to the peak shape and improved current value. Moreover, cycle number of Pd deposition was varied between 5 to 20 (Fig. 3b). Dependence of cycle number on the ORR peak currents were presented with column graph in Fig. 3d. According to the graph, optimum value was chosen as 15 for further studies. 


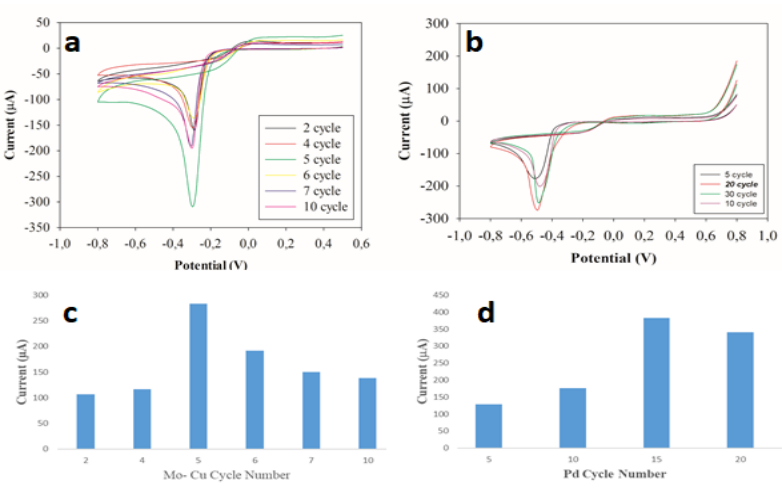

Figure 3. CVs of GCEs modified by electrodeposition of a) $\mathrm{CuOx}-\mathrm{MoOx}$ and b) $\mathrm{Pd}$ nanoparticles with various cycle numbers in $0.1 \mathrm{M} \mathrm{NaOH}$ at a scan rate of $50 \mathrm{mVs}^{-1}$. Dependence of peak current for ORR on the cycle number used during electrodeposition of c) $\mathrm{CuOx}$ MoOx and d) Pd nanoparticles onto GCE surface.

Comparative cyclic voltammograms of bare GCE, GCE/Pd, GCE/MoOx-CuOx and GCE/MoOx-CuOx/Pd were exhibited in Fig.4. Sluggish reduction peak at more negative potentials obtained for bare GCE and GCE/MoOx-CuOx. Individual presence of $\mathrm{Pd}$ nanoparticles improves the peak characteristics both for potential shift to positive values and current enhancement $(200 \mu \mathrm{A})$. The best catalytic activity towards ORR was achieved with GCE/MoOx-CuOx/Pd in terms of both peak current $(600 \mu \mathrm{A})$ and peak potential as compared to others.

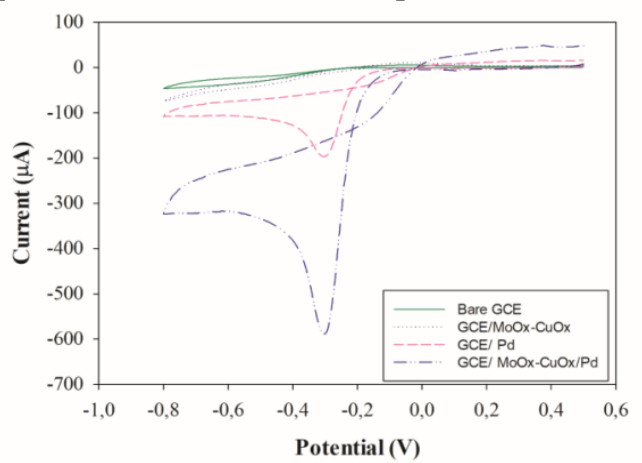

Figure 4. CVs of bare and various modified electrodes towards ORR in $0.1 \mathrm{M} \mathrm{NaOH}$.

All over the results are indicating that the developed electrode is good candidate for fuel cell applications due to the lower ORR potential with high current enhancement. The synergistic effect of metal oxides and palladium nanoparticles not only provided higher peak current but also provide positive potential shift. Since the ORR potential is very important issue for electrocatalysis, the ORR potential is compared to the proper literatures. Xiao et al. used Ag nanoparticle decorated oxygen-doped carbonaceous polypyrrole nanotubes for ORR and the peak potential was found about $-0.25 \mathrm{~V}$ [20], Pd nanoparticles supported on zeolite-templated mesocellular graphene was used as an electrocatlytic surface for ORR and reduction potential was found $-0.2 \mathrm{~V}$ [21], and in another study spin coated
Pd nanoparticles was used for ORR and reduction potential was found as $-0.3 \mathrm{~V}$ [22]. The ORR potential was found as $-0.25 \mathrm{~V}$ for $\mathrm{GCE} / \mathrm{MoOx}-\mathrm{CuOx} / \mathrm{Pd}$ electrode and this value is also compatible with literature values. The proposed electrode exhibited superior catalytic activity towards ORR since small amount of Pd loaded onto the electrode. In addition, electrochemical deposition provides a simple, low-cost and green methodology for the preparation of mixed oxide and Pd nanoparticles.

\section{Conclusion}

Here, GCE/MoOx-CuOx/Pd was fabricated with simple electrochemical technique with two steps that each step carry out with consecutive potential cycles. The resulting electrode characterized with SEM and existence of each component on the surface was proved with EDX results. The proposed composite $\mathrm{GCE} / \mathrm{MoOx}-\mathrm{CuOx} / \mathrm{Pd}$ was used as an efficient surface for electrocatalytic oxygen reduction which significantly improves the peak current of ORR as compared to the other bare and modified electrodes.

\section{Acknowledgement}

Author would like to thank for Dr. Çağrı Ceylan Koçak, and Prof. Dr. Nil Ertaş for their valuable contributions. This study was supported by EBYU Scientific Council with a project number of FHD-2018-552.

\section{Ethics}

There are no ethical issues after the publication of this manuscript.

\section{References}

1. Bakır ÇC, Şahin N, Polat R, Dursun Z. 2011. Electrocatalytic reduction of oxygen on bimetallic copper-gold nanoparticlesmultiwalled carbon nanotube modified glassy carbon electrode in alkaline solution. J. Electroanal. Chem; 662: 275-280.

2. Yavuz E, Özdokur KV, Çakar İ, Koçak S, Ertaş FN. 2015. Electrochemical preparation, characterization of molybdenumoxide/ platinum binary catalysts and its application to oxygen reduction reaction in weakly acidic medium. Electrochimica Acta; 151: $72-80$

3. Li X, Liu J, Du F, Peng H, Jiang L. 2019. Cations promoting synthesis of self-supported nanoporous silver electrode and its catalytic activity for oxygen reduction reaction. Applied Surface Science; 464: 21-29.

4. Garlyyev B, Pohl MD, Čolić V, Liang Y, Butt FK, Holleitner A, Bandarenka AS. 2018. High oxygen reduction reaction activity of Pt5Pr electrodes in acidic media. Electrochemistry Communications; 88: 10-14.

5. Koçak ÇC, Karabiberoğlu Ş. 2018. Electrochemical vanillin determination on gold nanoparticles modified multiwalled carbon nanotube electrode. Dokuz Eylul University-Faculty of Engineering Journal of Science and Engineering; 20: 59.

6. Sunil TS, Naik K, Mwaurah MM, Swamy BEK. 2019. Fabrication of poly (sudan III) modified carbon paste electrode sensor for dopamine: A voltammetric study. Journal of Electroanalytical Chemistry; 834: 71-78. 
7. Koçak ÇC, Dursun Z. 2018. Enhanced electrocatalytic activity of copper phthalocyanine/multiwalled carbon nanotube composite electrode via Pt nanoparticle modification for oxygen reduction. Turkish Journal of Chemistry; 42: 623- 638.

8. Wei W, Wu S-G. 2019. Study of Electrooxidation Behavior of Nitrite on Gold Nanoparticles/Graphitizing Carbon Felt Electrode and Its Analytical Application. Chinese Journal of Analytical Chemistry; 47: 19014-19020.

9. Çakar İ, Özdokur KV, Demir B, Yavuz E, Demirkol DO, Koçak S, Timur S, Ertaş FN. 2013. Molybdenum oxide/platinum modified glassy carbon electrode: A novel electrocatalytic platform for the monitoring of electrochemical reduction of oxygen and its biosensing applications. Sensors and Actuators B; 185: $331-336$.

10. Sunder GSS, Rohanifar A, Devasurendra AM, Kirchhoff JR 2019. Selective determination of 1-DOPA at a graphene oxide/yttrium oxide modified glassy carbon electrode. Electrochimica Acta; 301: 192-199.

11. Karim-Nezhad G, Khorablou Z, Zamani M, Dorraji PS Alamgholiloo M. 2017. Voltammetric sensor for tartrazine determination in soft drinks using poly (p-aminobenzenesulfonic acid)/zinc oxide nanoparticles in carbon paste electrode. Journal of Food and Drug Analysis; 25: 293-301.

12. Swetha JV, Parse H, Kakade B, Geetha A. 2018. Morphology dependent facile synthesis of manganese oxide nanostructures for oxygen reduction reaction. Solid State Ionics; 328: 1-7.

13. Zhang $\mathrm{T}$, Jin $\mathrm{H}$, Fang $\mathrm{Y}$, Guan JB, Ma SJ, Pan Y, Zhang M, Zhu H, Liu XD, Du ML. 2019. Detection of trace $\mathrm{Cd}^{2+}, \mathrm{Pb}^{2+}$ and $\mathrm{Cu}^{2+}$ ions via porous activated carbon supported palladium nanoparticles modified electrodes using SWASV. Materials Chemistry and Physics; 225: 433-442.

14. Shahvandi SK, Ahmar H, S. Rezaei JT. 2019. Palladium nanoparticles immobilized on polymer-functionalized magnetic nanoparticles for the determination of hydrogen peroxide. Surfaces and Interfaces; 12: 71-77.
15. Absalan G, Akhond M, Soleimani M, Ershadifar H. 2016. Efficient electrocatalytic oxidation and determination of isoniazid on carbon ionic liquid electrode modified with electrodeposited palladium nanoparticles. Journal of Electroanalytical Chemistry; 761: 1-7.

16. Karabiberoğlu Ş, Koçak ÇC. 2018. Voltammetric determination of vanillin in commercial food products using overoxidized poly(pyrrole) film-modified glassy carbon electrodes. Turkish Journal of Chemistry; 42: 291-305.

17. Koçak S., Ertaş F.N., Dursun Z., 2013. Electrochemical deposition and behavior of mixed-valent molybdenum oxide film at glassy carbon and ITO electrodes. Applied Surface Science; 265: $205-213$

18. Darko G., Batric P., 2002. Electrodeposition of copper: the nucleation mechanisms. Electrochimica Acta; 47: 2901-2912.

19. Byung-Kwon K., Daeha S., Ji Young L., Hyunjoon S., Juhyoun K. 2010. Electrochemical deposition of Pd nanoparticles on indium-tin oxide electrodes andtheir catalytic properties for formic acid oxidation. Electrochemistry Communications; 12: 1442-1445.

20. Xiao D, Ma J, Chen C, Luo Q, Ma J, Zheng L, Zuo X. 2018. Oxygen-doped carbonaceous polypyrrole nanotubes-supported $\mathrm{Ag}$ nanoparticle as electrocatalyst for oxygen reduction reaction in alkaline solution. Materials Research Bulletin; 105: 184-191.

21. Cui X, Xu Y, Chen L, Zhao M, Yanga S, Wang Y. 2019. Ultrafine Pd nanoparticles supported on zeolite-templated mesocellular graphene network via framework aluminum mediation: An advanced oxygen reduction electrocatalyst. Applied Catalysis B: Environmental; 244: 957-964.

22. Ansari AA, Sartale SD. 2015. Effect of processing parameters on size, density and oxygen reduction reaction (ORR) activity of Pd nanoparticles grown by spin coating. Surface \& Coatings Technology; 281: 68-75. 